complex (R), AHL (A) and a fixed concentration of LuxR. The following equations were used:

$$
\begin{gathered}
\frac{\mathrm{d} G}{\mathrm{~d} t}=\frac{\alpha_{\mathrm{G}}}{1+\left(L / \beta_{\mathrm{L}}\right)^{\eta 1}}-\gamma_{\mathrm{G}} G \\
\frac{\mathrm{d} L}{\mathrm{~d} t}=\frac{\alpha_{\mathrm{L} 1}}{1+\left(C / \beta_{\mathrm{C}}\right)^{\eta 2}}+\frac{\alpha_{\mathrm{L} 2} \cdot R^{\eta 3}}{\left(\theta_{\mathrm{R}}\right)^{\eta 3}+R^{\eta 3}}-\gamma_{\mathrm{L}} L \\
\frac{\mathrm{d} C}{\mathrm{~d} t}=\frac{\alpha_{\mathrm{C}} R^{\eta 3}}{\left(\theta_{\mathrm{R}}\right)^{\eta 3}+R^{\eta 3}}-\gamma_{\mathrm{C}} C \\
\frac{\mathrm{d} R}{\mathrm{~d} t}=\rho_{\mathrm{R}}[\operatorname{LuxR}]^{2} A^{2}-\gamma_{\mathrm{R}} R \\
\frac{\mathrm{d} A_{x, y, z}=\xi\left(A_{x-1, y, z}+A_{x+1, y, z}+A_{x, y-1, z}+A_{x, y+1, z}+A_{x, y, z-1}+A_{x, y, z+1}-6 A_{x, y, z}\right)}{\mathrm{d} t}-\gamma_{\mathrm{A}}
\end{gathered}
$$

The following parameters approximate the behaviour of BD2: protein synthesis rates $\left(\alpha_{\mathrm{G}}\right.$, $\left.2 \mu \mathrm{M} \mathrm{min}^{-1} ; \alpha_{\mathrm{L} 1}, 1 \mu \mathrm{M} \mathrm{min}^{-1} ; \alpha_{\mathrm{L} 2}, 1 \mu \mathrm{M} \mathrm{min}^{-1} ; \alpha_{\mathrm{C}}, 1 \mu \mathrm{M} \mathrm{min}^{-1}\right)$, repression coefficients $\left(\beta_{\mathrm{L}}, 0.8 \mu \mathrm{M} ; \beta_{\mathrm{C}}, 0.008 \mu \mathrm{M}\right)$, protein decay $\left(\gamma_{\mathrm{C}}\right.$ and $\gamma_{\mathrm{G}}, 0.0692 \mathrm{~min}^{-1} ; \gamma_{\mathrm{L}}$ and $\left.\gamma_{\mathrm{R}}, 0.0231 \mathrm{~min}^{-1}\right)$, LuxR/AHL activation coefficient $\left(\theta_{\mathrm{R}}, 0.01 \mu \mathrm{M}\right)$, transcription factor cooperativity/multimerization $\left(\eta_{1}, 2 ; \eta_{2}, 2 ; \eta_{3}, 1\right)$, LuxR/AHL dimerization $\left(\rho_{\mathrm{R}}\right.$,

$\left.0.5 \mu \mathrm{M}^{-3} \mathrm{~min}^{-1}\right)$, AHL intercellular diffusion $\left(\xi, 0.001 \mathrm{~mm}^{2} \mathrm{~min}^{-1}\right)$ and AHL decay $\left(\gamma_{\mathrm{A}}, 0.01 \mathrm{~min}^{-1}\right)$. LuxR concentration was set at $0.5 \mu \mathrm{M}$. LuxR and AHL first bind to form a complex, and this complex then dimerizes to form an active transcription factor ${ }^{17}$; hence the quadratic terms in equation (4). The degradation rate of AHL $\left(\gamma_{\mathrm{A}}\right)$, which determines the steepness of the chemical gradient, is affected by $\mathrm{pH}$ (ref. 18). The liquid-phase simulations did not include equation (5). Further, to simulate the high-detect component in the liquid phase, equation (3) and CI repression in equation (2) were also not used. For the HD1 and BD1 liquid simulations, $\rho_{\mathrm{R}}$ was increased tenfold from above, whereas for the HD3 and BD3 simulations the values of $\alpha_{\mathrm{G}}, \alpha_{\mathrm{L} 2}$ and [LuxR] were halved.

For the statistical analysis of the shift, we first generated 2,000 sets of random kinetic parameters, in which the values for each parameter were uniformly distributed around those used above. Among these sets, about 30\% yielded band-detect behaviour with a gain greater than three and fluorescence values above a predetermined threshold. For the first 100 sets we computed the AHL synthesis rate resulting in ring formation centred at $7 \mathrm{~mm}$ from the senders. Using each parameter set, we simulated system behaviour until GFP at all positions stabilized, and computed the shift as follows. The beginning of a shift is defined as the spatiotemporal coordinate $<$ position $_{\text {start }}$, time $_{\text {start }}>$ that meets the following criteria: it is in the middle of the ring for that particular time, it is closest to the origin, and its fluorescence is greater than $50 \%$ of maximum overall steady-state fluorescence. The end of a shift is defined as the spatiotemporal coordinate

$<$ position $_{\text {steady }}$ time steady $>$ that meets the same criteria as above and is located at the final steady-state position of the ring. The positional shift is then the difference between position $_{\text {steady }}$ and position start $_{\text {. }}$

In Fig. $4 \mathrm{~b}, \mathrm{c}$ the following values were changed from above $\left(\beta_{\mathrm{L}}, 543 / 230 \mathrm{nM} ; \beta_{\mathrm{C}}, 19\right.$ / $69 \mathrm{nM} ; \gamma_{C}, 0.116 / 0.496 \mathrm{~min}^{-1} ; \gamma_{\mathrm{G}}, 0.404 / 0.366 \mathrm{~min}^{-1} ; \gamma_{\mathrm{L}}, 0.018 / 0.902 \mathrm{~min}^{-1} ; \gamma_{\mathrm{R}}$, $\left.0.036 / 0.050 \mathrm{~min}^{-1} ; \theta_{\mathrm{R}}, 0.249 / 0.063 \mu \mathrm{M} ; \rho_{\mathrm{R}}, 2.102 / 3.374 \mu \mathrm{M}^{-3} \mathrm{~min}^{-1}\right)$. GraphPad PRISM software was used to perform a regression analysis correlating each of the rate constants with the corresponding shift values. The response times were correlated by using powerseries equations and the positional shift was correlated with a sigmoidal fit.

Received 3 December 2004; accepted 14 February 2005; doi:10.1038/nature03461.

1. Golden, J. \& Yoon, H. Heterocyst formation in anabaena. Curr. Opin. Microbiol. 1, 623-629 (1998).

2. Scherrer, R. \& Shull, V. Structure, partial elemental composition, and size of Thiopedia rosea cells and platelets. Can. J. Microbiol. 32, 607-610 (1986).

3. Ben-Jacob, E. et al. Cooperative formation of chiral patterns during growth of bacterial colonies. Phys. Rev. Lett. 75, 2899-2902 (1995)

4. Fuqua, W. C., Winans, S. \& Greenberg, E. P. Quorum sensing in bacteria: The luxR-luxI family of cell density-responsive transcriptional regulators. J. Bacteriol. 1760, 269-275 (1994).

5. Bassler, B. L. How bacteria talk to each other: regulation of gene expression by quorum sensing. Curr. Opin. Microbiol. 2, 582-587 (1999).

6. Milo, R. et al. Network motifs: simple building blocks of complex networks. Science 298, 824-827 (2002).

7. Collins, C. H., Arnold, F. H. \& Leadbetter, J. R. Directed evolution of Vibrio fischeri LuxR for increased sensitivity to a broad spectrum of acyl-homoserine lactones. Mol. Microbiol. 55, 712-723 (2005).

8. Jaeger, J. et al. Dynamical analysis of regulatory interactions in the gap gene system of Drosophila melanogaster. Genetics 167, 1721-1737 (2004).

9. Jaeger, J. et al. Dynamic control of positional information in the early Drosophila embryo. Nature $\mathbf{4 3 0}$, 368-371 (2004)

10. Weiss, R. \& Knight, T. F. Jr in DNA6: Sixth International Workshop on DNA-Based Computers, DNA2000 (eds Condon, A. \& Rozenberg, G.) 1-16 (Springer, Leiden, The Netherlands, 2000).

11. Ptashne, M. A Genetic Switch: Phage Lambda and Higher Organisms, 2nd edn (Cell Press and Blackwell Scientific Publications, Cambridge, Massachusetts, 1986).

12. Egland, K. A. \& Greenberg, E. P. Quorum sensing in Vibrio fischeri: elements of the luxI promoter. Mol. Microbiol. 31, 1197-1204 (1999).

13. Weiss, R. \& Basu, S. in NSC-1: The First Workshop of Non Silicon Computing 〈http://www2.cs.cmu.edu/ phoenix/nsc1/paper/3-2.pdf $\rangle$ (2002).

14. Basu, S., Mehreja, R., Thiberge, S., Chen, M. \& Weiss, R. Spatiotemporal control of gene expression with pulse-generating networks. Proc. Natl Acad. Sci. USA 101, 6355-6360 (2004).

15. Weiss, R. Cellular Computation and Communication Using Engineered Genetic Regulatory Networks. Ph.D. thesis, Massachusetts Inst. Technology (2001).
16. Andersen, J. B. et al. New unstable variants of green fluorescent protein for studies of transient gene expression in bacteria. Appl. Environ. Microbiol. 64, 2240-2246 (1998).

17. Choi, S. H. \& Greenberg, E. P. Genetic evidence for multimerization of LuxR, the transcriptional activator of Vibrio fischeri luminescence. Mol. Mar. Biol. Biotechnol. 6, 408-413 (1992).

18. Yates, E. A. et al. $\mathrm{N}$-acylhomoserine lactones undergo lactonolysis in a $\mathrm{pH}-$, temperature-, and acyl chain length-dependent manner during growth of Yersinia pseudotuberculosis and Pseudomonas aeruginosa. Infect. Immun. 70, 5635-5646 (2002).

Supplementary Information accompanies the paper on www.nature.com/nature.

Acknowledgements We thank D. Karig, S. Hooshangi, S. Thiberge, M.-T. Chen and S. Subramaniam for discussions or comments on the manuscript. This material is based on work supported by the Defense Advanced Research Projects Agency (DARPA).

Competing interests statement The authors declare that they have no competing financial interests.

Correspondence and requests for materials should be addressed to R.W. (rweiss@princeton.edu).

\section{Dynamics of Drosophila embryonic patteming network perturbed in space and time using microfluidics}

\section{Elena M. Lucchetta ${ }^{1}$, Ji Hwan Lee ${ }^{1}$, Lydia A. Fu ${ }^{1}$, Nipam H. Patel \& Rustem F. Ismagilov}

${ }^{1}$ Department of Chemistry, University of Chicago, Chicago, Illinois 60637, USA ${ }^{2}$ Department of Integrative Biology, Molecular and Cell Biology, and Howard Hughes Medical Institute, University of California Berkeley, Berkeley, California 94720-3140, USA

Biochemical networks are perturbed both by fluctuations in environmental conditions and genetic variation. These perturbations must be compensated for, especially when they occur during embryonic pattern formation. Complex chemical reaction networks displaying spatiotemporal dynamics have been controlled and understood by perturbing their environment in space and time $\mathrm{i}^{1-3}$. Here, we apply this approach using microfluidics to investigate the robust network in Drosophila melanogaster that compensates for variation in the Bicoid morphogen gradient. We show that the compensation system can counteract the effects of extremely unnatural environmental conditions-a temperature step_in which the anterior and posterior halves of the embryo are developing at different temperatures and thus at different rates. Embryonic patterning was normal under this condition, suggesting that a simple reciprocal gradient system is not the mechanism of compensation. Time-specific reversals of the temperature step narrowed down the critical period for compensation to between 65 and $100 \mathrm{~min}$ after onset of embryonic development. The microfluidic technology used here may prove useful to future studies, as it allows spatial and temporal regulation of embryonic development.

Although rates of production and degradation of morphogens are affected by genetic and environmental variations, the mechanisms of embryo patterning have evolved to compensate for these variations. In Drosophila melanogaster, morphogens such as Bicoid protein act early on in the genetic hierarchy that patterns the Drosophila embryo along the antero-posterior axis. Altering the copy number of bicoid genes shifts the Bicoid expression profile and results in shifting of the expression pattern of a direct downstream target gene, hunchback, and subsequent expression of pair-rule and segment polarity genes ${ }^{4}$. The expression profile of the maternal Bicoid protein gradient, however, varies measurably between individual wild-type embryos, and is particularly affected by variations 
in temperature ${ }^{5}$. This naturally occurring variation does not result in altered expression of hunchback ${ }^{5}$, suggesting that there must be a compensatory mechanism to filter noise from the initial maternal Bicoid gradient. This compensation is important because the correct expression of gap and pair-rule genes is required to relay positional information for determining cell fate, although later compensation for shifts in patterning is also possible ${ }^{4}$. It has recently been suggested that anterior shifts in gap gene expression may be due to dynamic establishment of the Bicoid gradient in addition to regulation among the gap genes ${ }^{6}$. However, even in these analyses, the Hunchback protein boundary was stable ${ }^{6}$.

Although the exact nature of compensation is unknown, we wanted to test the limits of compensation, to determine whether compensation occurs by a simple reciprocal posterior gradient system, and to determine precisely when the compensatory mechanism functions during early development. We developed a microfluidic apparatus to precisely control the temperature of different parts of the embryo both spatially and temporally, thus differentially controlling the rate of development in the anterior and posterior halves of the embryo. In the natural world, a developing Drosophila embryo is unlikely to experience conditions that maintain the two halves of the embryo at different temperatures. Thermal diffusion on the scale of a Drosophila embryo $(500 \mu \mathrm{m})$ is rapid and would equalize the temperatures in the embryo's environment and the two halves of the embryo within seconds.

We used microfluidic laminar flow ${ }^{7-10}$ to create temperature differences by flowing two converging aqueous streams around an embryo, each at a controlled temperature, to provide rapid supply and removal of heat. The apparatus consists of two asymmetric moulds with alignment posts and holes ${ }^{11}$; moulds were made in polydimethylsiloxane (PDMS) ${ }^{12}$, which has low thermal conductivity. The microfluidic device can be assembled in one minute around a live embryo, with the result that the embryo is suspended in the cross-section of the channel (Fig. 1a) (see Supplementary Information for details on microfabrication, assembly of the microfluidic device and characterization of the flow).

We first demonstrated that development of the embryo was not adversely affected by flow at uniform temperature. Shear rate at the embryo was $\sim 700 \mathrm{~s}^{-1}$, which was within biological range $\mathrm{e}^{13}$, and dissolved oxygen in the flow was within the range of normoxia ${ }^{14}$. Embryos kept under flow in control experiments, where both streams were $20^{\circ} \mathrm{C}$ or both streams were $27^{\circ} \mathrm{C}$, developed normally (see Supplementary Information).

A temperature step ( $T$-step) was created by separately heating one laminar stream of flow and cooling the other. The difference in temperature was visualized using thermochromic liquid crystals ${ }^{15}$ (Fig. 1b). The response time ${ }^{15}$ of thermochromic liquid crystals is

a

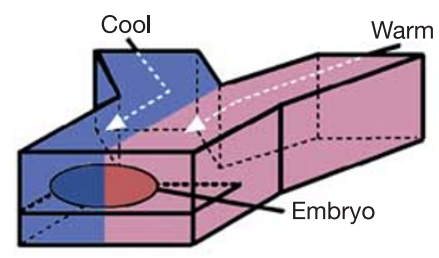

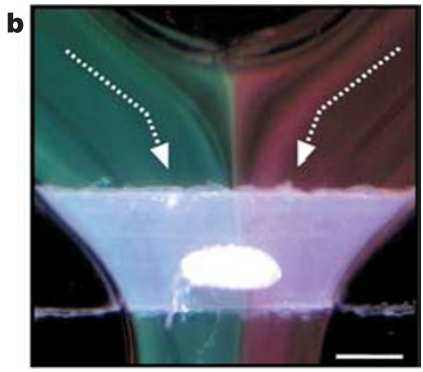

Figure 1 Experimental set up. a, A schematic drawing of a PDMS microfluidic device with a $D$. melanogaster embryo developing in a temperature-step ( $T$-step). b, A microphotograph illustrating the $T$-step around the embryo, visualized using a suspension of thermochromic liquid crystals (Image Therm Engineering) flowing at a flow rate of $50 \mathrm{~mm} \mathrm{~s}^{-1}$. The temperature of the green stream is $21^{\circ} \mathrm{C}$ and the temperature of the red stream is $24^{\circ} \mathrm{C}$. Scale bar, $400 \mu \mathrm{m}$. $\sim 10 \mathrm{~ms}$, and thus the colour at a given point in the channel is representative of the temperature $500 \mu \mathrm{m}$ upstream of that point. Across the embryo, thermal diffusion between the two streams was calculated to be $\sim 50 \mu \mathrm{m}$, which corresponds to $\sim 10 \%$ of the egg length (EL). We calculated that the timescale of heat transfer was $\sim 10 \mu$ s through the $\sim 1.5-\mu \mathrm{m}$ thick eggshell, much shorter than the timescale of heat transfer of $\sim 1 \mathrm{~s}$ across the $\sim 500-\mu \mathrm{m}$ long embryo (see Supplementary Information).

Differences in the density of nuclei in the two halves of the embryo showed experimentally that development was affected by the $T$-step. In normal embryonic development, 13 cycles of nuclear division are followed by cellularization of the embryo during the fourteenth cycle $^{16}$. Although there are transient waves of mitosis, the cycles occur nearly simultaneously across the embryo and the density of nuclei stays relatively uniform.

Embryos developing in a T-step had a higher density of nuclei in the warmer half, confirming that the warmer half was developing more rapidly (Fig. 2). Embryos showed a greater difference when exposed to a larger $T$-step for a longer time $\left(17^{\circ} \mathrm{C} / 27^{\circ} \mathrm{C}\right.$ for $150 \mathrm{~min}$; Fig. 2c, d) than when exposed to a smaller $T$-step for a shorter time $\left(20^{\circ} \mathrm{C} / 27^{\circ} \mathrm{C}\right.$ for $140 \mathrm{~min}$; Fig. 2a, b). Embryos exposed to a $T$-step of $17^{\circ} \mathrm{C} / 27^{\circ} \mathrm{C}$ showed a difference of two cell cycles (a fourfold difference in nuclear density) between the anterior and posterior halves, with the cool half closest to cycle 11 and the warm

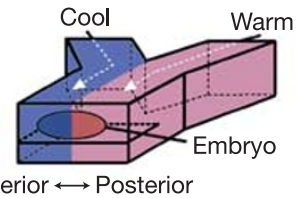

Anterior $\longleftrightarrow$ Posterior
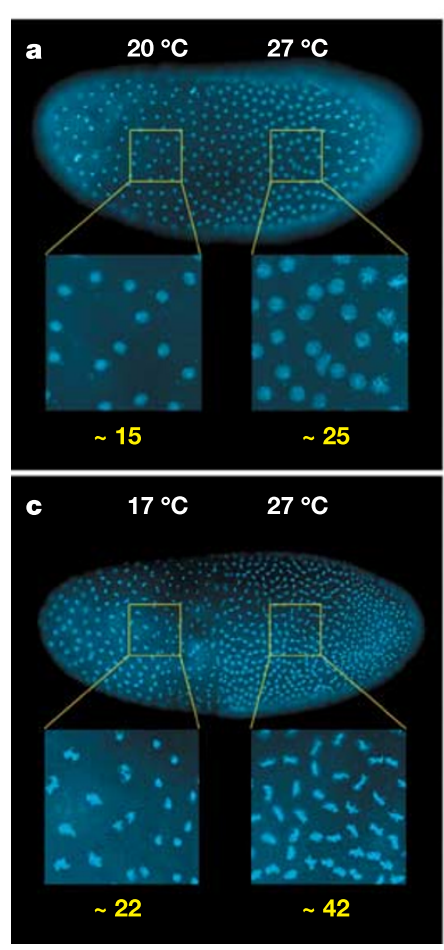

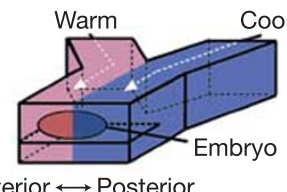

Anterior $\longleftrightarrow$ Posterior
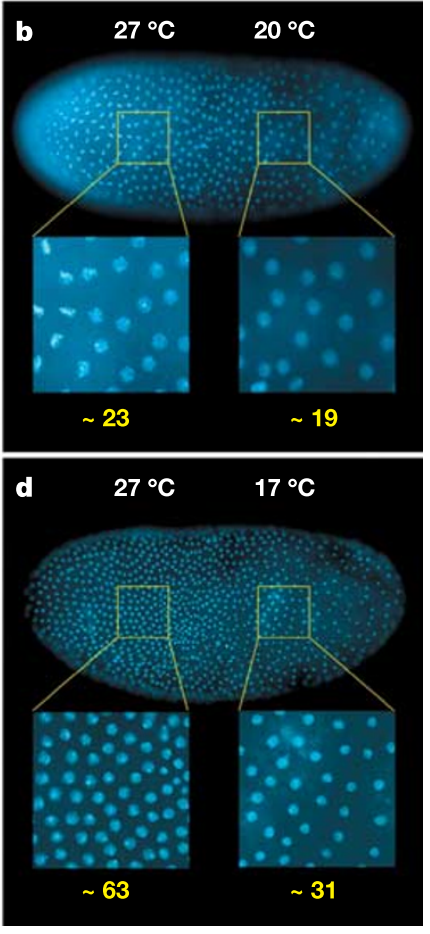

Figure 2 The rate of development in each half of the embryo exposed to a T-step is affected by temperature. Each half of the embryo is in a different cell cycle, as demonstrated by difference in nuclear density. Number of nuclei in enlarged areas shown underneath in yellow numbering. a, b, Embryos exposed to a $T$-step of $20^{\circ} \mathrm{C} / 27^{\circ} \mathrm{C}$ for $140 \mathrm{~min}$. a, Anterior half $20^{\circ} \mathrm{C}$, posterior half $27^{\circ} \mathrm{C}$. b. Anterior half $27^{\circ} \mathrm{C}$, posterior half $20^{\circ} \mathrm{C}$. c, d, Embryos exposed to a $T$-step of $17^{\circ} \mathrm{C} / 27^{\circ} \mathrm{C}$ for $150 \mathrm{~min}$. c, Anterior half $17^{\circ} \mathrm{C}$, posterior half $27^{\circ} \mathrm{C}$. d, Anterior half $27^{\circ} \mathrm{C}$, posterior half $17^{\circ} \mathrm{C}$. In all images, higher nuclear density was observed in the warmer half of the embryo. 

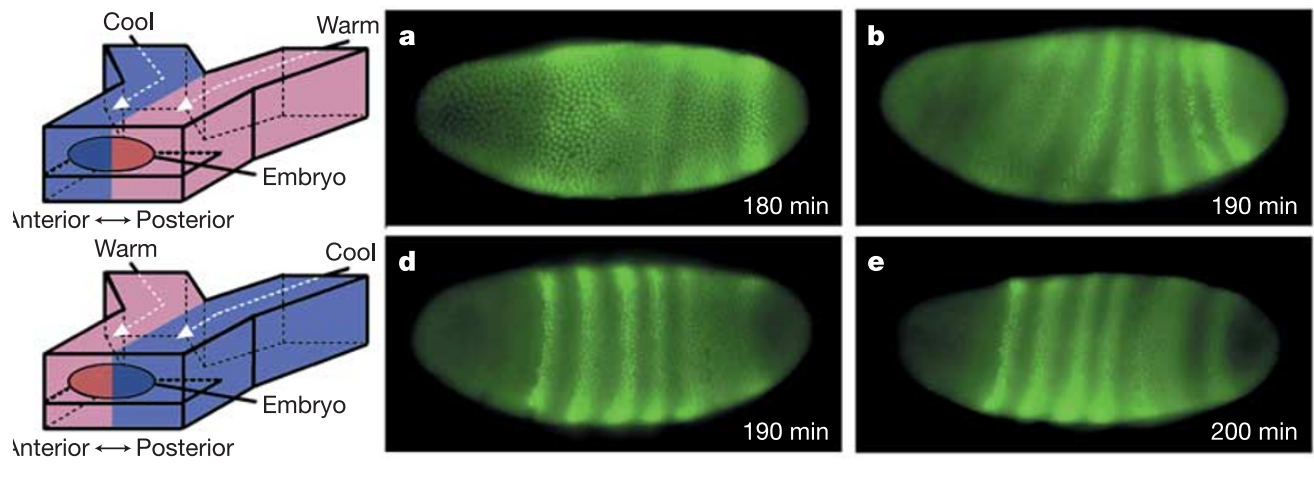
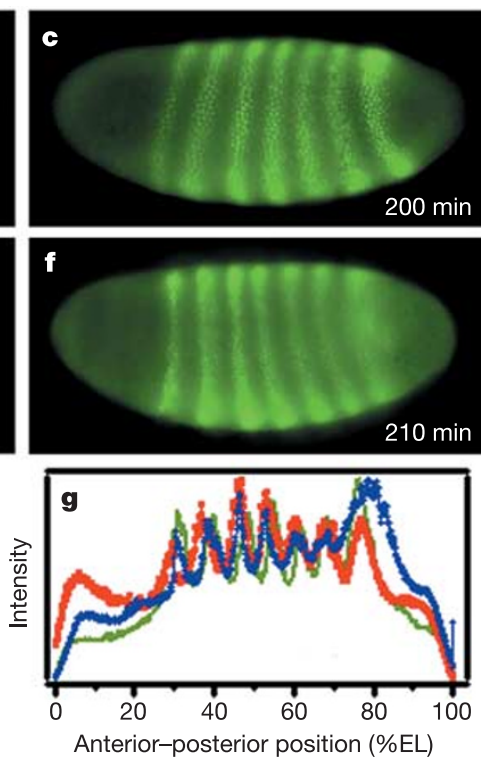

g, Intensity profile of Even-skipped expression in embryos exposed to the $T$-step in panels c (red) and $\mathbf{f}$ (blue), compared to an average intensity profile (green) of Even-skipped expression in four control embryos that developed at room temperature. a-c, Embryos with a cool anterior half and warm posterior half. $\mathbf{d}-\mathbf{f}$, Embryos with a warm anterior half and cool posterior half. Even-skipped stripes were consistently expressed in the warm half first (a and $\mathbf{b}, \mathbf{d}$ and $\mathbf{e}$ ), but resolved in the correct positions $(\mathbf{c}, \mathbf{f})$. half closest to cycle 13 in control embryos. Therefore, the difference in nuclear density was more than a mitotic wave. Embryos exposed to a T-step of $20^{\circ} \mathrm{C} / 27^{\circ} \mathrm{C}$ showed a difference of one cell cycle (a twofold difference in nuclear density) between the anterior and posterior halves, with the cool half and warm half closest to cycle 11 and 12 in controls, respectively (see Supplementary Information). Notably, the boundary in nuclear density was relatively sharp, not only confirming a tight thermal boundary, but demonstrating that cell cycle regulation could occur fairly independently between neighbouring nuclei in the syncytial blastoderm. Wasp embryos set in an agar block over heating elements have previously been exposed to temperature gradients ${ }^{17}$. Notably, these experiments resulted in normal patterning late in development, but as no molecular markers were assayed, it was unclear at what point compensation occurred. Furthermore, the morphogen gradients involved in patterning in wasps are unknown.

Our initial presumption was that Drosophila embryos developing in the unusual environment established within our microfluidic apparatus would have obvious defects in patterning. It has been shown that the shape of the Bicoid gradient, which is determined by a combination of production, degradation and diffusion of Bicoid, is strongly affected by temperature ${ }^{5}$. In our experiments, these processes will occur at different rates in the two halves of embryos. To our surprise, embryos allowed to develop in a temperature step (either $17^{\circ} \mathrm{C} / 27^{\circ} \mathrm{C}$ or $20^{\circ} \mathrm{C} / 27^{\circ} \mathrm{C}$ ) for $150 \mathrm{~min}$ and then left at room temperature developed into normal larvae with the correct number and pattern of segments (data not shown). The normal appearance of larvae suggests that the embryo compensates for the unnatural temperature environment.

We used the microfluidic system to test whether patterning of Even-skipped ${ }^{18}$ remained robust in embryos exposed to the T-step. Normally, Even-skipped resolves into seven stripes in a specific order. First, stripes 1 and 2 resolve, and then stripe 7 resolves. Stripes $3,4,5$, and 6 resolve next, more or less simultaneously ${ }^{18}$. Once resolved (by late cycle 14), the stripes are positioned at $\sim 31,40,48$, 55, 62, 69, and 78\% EL ${ }^{19}$. The pattern of Even-skipped expression was monitored at the T-step of $20^{\circ} \mathrm{C} / 27^{\circ} \mathrm{C}$ (Fig. 3), which was chosen so that we could observe the entire embryo within cycle 14 . A temperature difference greater than this causes gastrulation and germband extension to begin in the warmer half before Evenskipped stripes in the cooler half resolve (see Supplementary Information), making measurement of stripe positions inaccurate, but illustrating that the two halves still develop at different rates. Embryos exposed to the T-step $\left(20^{\circ} \mathrm{C} / 27^{\circ} \mathrm{C}\right)$ clearly formed Evenskipped stripes out of order. When the anterior half of the embryo was warmer, Even-skipped stripes 1, 2 and 3 consistently resolved first (Fig. 3d, e). When the posterior half of the embryo was warmer, Even-skipped stripes 5, 6 and 7 consistently resolved first (Fig. 3a, b); this was particularly striking because in normal development stripes 5 and 6 resolve last.

Surprisingly, Even-skipped stripe positions were precise, despite the difference in developmental rate in the two halves of the embryo (Fig. 3c, f). Even-skipped stripes 1 through 7 resolved at 30, 38, 48, $54,62,68$, and $78 \pm 1 \% \mathrm{EL}$, respectively, for embryos exposed to a T-step of $20^{\circ} \mathrm{C} / 27^{\circ} \mathrm{C}$ (with either half being warmer) (Fig. $3 \mathrm{~g}$ ). Embryos exposed to the $T$-step of $20^{\circ} \mathrm{C} / 27^{\circ} \mathrm{C}$ also compensated for the unnatural environment at the level of Hunchback expression, which is a more direct readout of the maternal Bicoid morphogen gradient. For embryos with anterior $27^{\circ} \mathrm{C}$ /posterior $20^{\circ} \mathrm{C}$ (Fig. 4a) and embryos with anterior $20^{\circ} \mathrm{C} /$ posterior $27^{\circ} \mathrm{C}$ (data not shown), the Hunchback boundary remained spatially precise (46-51\% EL), comparable to control embryos ${ }^{5}$.

We determined the critical period during which the compensation mechanism functions by setting up a temperature regimen as follows: embryos developed with anterior at $27^{\circ} \mathrm{C} /$ posterior at $20^{\circ} \mathrm{C}$ with the exception of a brief $35-$ min period during which the orientation of the $T$-step was transiently reversed (anterior $20^{\circ} \mathrm{C} /$ posterior $27^{\circ} \mathrm{C}$ at $t_{1}$ ) before being reversed back (anterior $27^{\circ} \mathrm{C} /$ posterior $20^{\circ} \mathrm{C}$ at $t_{2}$ ). Embryos exposed to a brief temperature reversal $\left(t_{1}=65 \mathrm{~min}\right.$ and $\left.t_{2}=100 \mathrm{~min}\right)$ showed a marked increase in variability of the Hunchback boundary (35-53\% EL) with anterior bias (Fig. 4b), resembling the embryos carrying the stau ${ }^{\mathrm{HL}}$ or the $\operatorname{stau}^{\mathrm{r} 9}$ allele $^{5}$, which also showed variability with anterior bias. Corresponding nuclear images stained with 4,6-diamidino-2phenylindole (DAPI) do not support the possibility that the increased variability of the Hunchback boundary position is simply due to differences in the ages of the embryos in cycle 14 (see Supplementary Information). Other earlier $\left(t_{1}=35 \mathrm{~min}\right.$, 


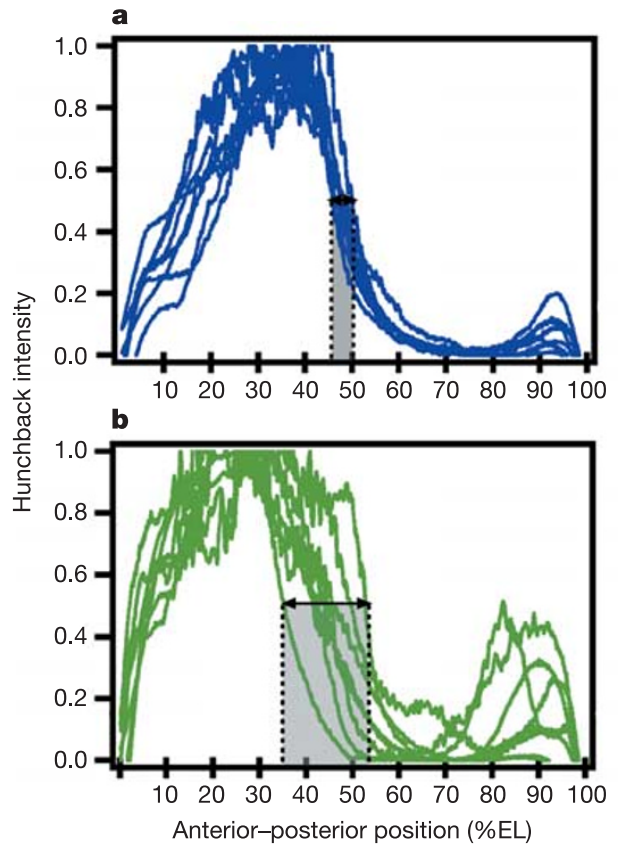

Figure 4 Hunchback expression in embryos exposed to a $T$-step and time-dependent $T$-step. a, Hunchback intensity profiles of embryos with anterior half at $27^{\circ} \mathrm{C}$ and posterior half at $20^{\circ} \mathrm{C}$. Hunchback position was normal, varying over $5 \%$ EL (46-51\% EL). b. Hunchback intensity profiles of embryos exposed to a time-dependent $T$-step with anterior half at $27^{\circ} \mathrm{C}$ and posterior half at $20^{\circ} \mathrm{C}$, with the exception of a brief temperature reversal (anterior $20^{\circ} \mathrm{C} /$ posterior $27^{\circ} \mathrm{C}$ ) between 65 and $100 \mathrm{~min}$. The position of Hunchback was variable over 18\% EL (35-53\% EL).

$\left.t_{2}=70 \mathrm{~min}\right)$ or later $\left(t_{1}=95 \mathrm{~min}, t_{2}=130 \mathrm{~min}\right)$ transient reversals of the $T$-step resulted in relatively low variability in the Hunchback boundary (42-49\% and $43-50 \%$ EL, respectively) (see Supplementary Information). These results show that a key part of the compensatory mechanism is established between 65 and $100 \mathrm{~min}$. By extension, we suggest that normally occurring variations are 'measured' and possibly compensated for during this stage of development.

To test the possible polarity of the correction mechanism, we inverted the temperature regimen: embryos developed with anterior at $20^{\circ} \mathrm{C}$ and posterior at $27^{\circ} \mathrm{C}$, with the exception of a brief $35-\mathrm{min}$ period during which the orientation of the $T$-step was transiently reversed (anterior $27^{\circ} \mathrm{C} /$ posterior $20^{\circ} \mathrm{C}$ at $t_{1}$ ) before being reversed back (anterior $20^{\circ} \mathrm{C} /$ posterior $27^{\circ} \mathrm{C}$ at $t_{2}$ ). Embryos exposed to later temperature reversals $\left(t_{1}=65 \mathrm{~min}, t_{2}=100 \mathrm{~min}\right.$ or $t_{1}=95 \mathrm{~min}$, $t_{2}=130 \mathrm{~min}$ ) showed low variability in the Hunchback boundary (43-48\% or $42-49 \%$ EL, respectively). Embryos exposed to an earlier temperature reversal $\left(t_{1}=35 \mathrm{~min}, t_{2}=70 \mathrm{~min}\right)$ also showed low variability in the Hunchback boundary (51-55\% EL), but in this case with a slight posterior bias (see Supplementary Information).

Using microfluidics, we changed the relative rate of development of the two halves of the embryo. Interestingly, Even-skipped expression in Fig. 3d and e bears resemblance to the pattern seen in beetle embryos ${ }^{20}$, in that Even-skipped stripes do not all appear more or less simultaneously. Despite our ability to change the temporal order in which Even-skipped stripes form, the spatial precision of the pattern remained intact. Thus, the low variability of the Hunchback boundary and Even-skipped pattern in these embryos suggests that the compensatory mechanism of the embryo can allow for correct patterning even in extremely unnatural environments.

A plausible compensatory mechanism for maintaining a stable
Hunchback boundary during normal development could involve an opposing gradient system using the anterior Bicoid gradient plus an unknown posterior gradient. Previous work has ruled out many genetic candidates for the possible posterior gradient (including nanos and caudal), but found that certain staufen alleles affected precision, suggesting that the compensatory system might involve the localization of an uncharacterized messenger RNA ${ }^{5}$. Our results suggest that the compensation system is not simply a reciprocal posterior gradient, as such a system would have failed when the two halves of the embryo developed at two different temperatures. Temperature reversal experiments lead us to suggest that the compensation mechanism has a critical establishment phase corresponding to $65-100 \mathrm{~min}$ of development.

Understanding the dynamics of a complex system by perturbing its environment in space and time does not require a priori knowledge of the system's components. This approach has been used to understand and control biological systems (such as appearance of fibrillations in a mammalian heart ${ }^{21}$ ) and chemical reaction networks (such as formation of patterns in the Belousov-Zhabotinsky reaction $^{3}$ and in surface-catalysed $\mathrm{CO}$ oxidations ${ }^{1}$ ). We believe that the microfluidic methods we have established here will prove to be an experimentally powerful approach that will allow the environment of the embryos to be spatiotemporally controlled. Perturbing the environment is a complementary approach to perturbing the molecular components of the network, as it might provide information on where and when events occur, rather than which molecules are involved. A combination of these two approaches might prove especially useful for identifying correction mechanisms responsible for the robustness of developmental and other biochemical networks.

\section{Methods}

Fly stocks, fabrication of the microfluidic device, and experimental set up All embryos were collected using wild-type Oregon R. Drosophila. Multilevel PDMS moulds were made using rapid prototyping. Embryos were collected at $23^{\circ} \mathrm{C}$ for $2-5 \mathrm{~min}$, placed on double-sided tape and assembled in the microfluidic device. Embryos were exposed to flow within $10 \mathrm{~min}$ of the start of collection. Syringe pumps (KdScientific) were used to control volumetric flow rate $\left(2 \mathrm{ml} \mathrm{min}^{-1}\right.$ total). Temperature of each laminar stream was maintained using a double chilling/heating plate (Echo therm, Torrey Pines Scientific).

\section{Immunostaining}

After removal from flow, embryos were dechorionated, fixed in 3\% formaldehyde in PEM buffer and immunostained using standard methods with anti-Even-skipped (mouse monoclonal 2B8) and anti-Hunchback (mouse monoclonal 1G10) antibodies, and goat anti-mouse IgG $(\mathrm{H}+\mathrm{L})$ AlexaFluor 488 conjugated secondary antibody (Molecular Probes).

\section{Image acquisition and analysis}

Images were acquired using a Leica DM IRE2 inverted microscope with a $\times 200.7 \mathrm{NA}$ objective and cooled CCD camera ORCA ERG 1394 (12-bit, $1344 \times 1024$ resolution, Hamamatsu Photonics). Even-skipped and Hunchback expression intensity profiles were analysed using MetaMorph Imaging System (Universal Imaging Corp).

See Supplementary Information for details regarding Methods.

Received 11 November 2004; accepted 23 February 2005; doi:10.1038/nature03509.

1. Wolff, J. et al. Spatiotemporal addressing of surface activity. Science 294, 134-137 (2001).

2. Sakurai, T., Mihaliuk, E., Chirila, F. \& Showalter, K. Design and control of wave propagation patterns in excitable media. Science 296, 2009-2012 (2002).

3. Vanag, V. K. et al. Oscillatory cluster patterns in a homogeneous chemical system with global feedback. Nature 406, 389-391 (2000).

4. Driever, W. \& Nusslein Volhard, C. The Bicoid protein determines position in the Drosophila embryo in a concentration-dependent manner. Cell 54, 95-104 (1988).

5. Houchmandzadeh, B., Wieschaus, E. \& Leibler, S. Establishment of developmental precision and proportions in the early Drosophila embryo. Nature 415, 798-802 (2002).

6. Jaeger, J. et al. Dynamic control of positional information in the early Drosophila embryo. Nature 430, 368-371 (2004).

7. Kenis, P. J. A., Ismagilov, R. F. \& Whitesides, G. M. Microfabrication inside capillaries using multiphase laminar flow patterning. Science 285, 83-85 (1999).

8. Takayama, S. et al. Subcellular positioning of small molecules. Nature 411, 1016 (2001)

9. Hatch, A. et al. A rapid diffusion immunoassay in a T-sensor. Nature Biotechnol. 19, 461-465 (2001).

10. Sia, S. K. \& Whitesides, G. M. Microfluidic devices fabricated in poly(dimethylsiloxane) for biological studies. Electrophoresis 24, 3563-3576 (2003).

11. Anderson, J. R. et al. Fabrication of topologically complex three-dimensional microfluidic systems in PDMS by rapid prototyping. Anal. Chem. 72, 3158-3164 (2000). 
12. McDonald, J. C. \& Whitesides, G. M. Poly(dimethylsiloxane) as a material for fabricating microfluidic devices. Accounts Chem. Res. 35, 491-499 (2002).

13. Furlong, E. E. M., Profitt, D. \& Scott, M. P. Automated sorting of live transgenic embryos. Nature Biotechnol. 19, 153-156 (2001).

14. DiGregorio, P. J., Ubersax, J. A. \& O'Farrell, P. H. Hypoxia and nitric oxide induce a rapid, reversible cell cycle arrest of the Drosophila syncytial divisions. J. Biol. Chem. 276, 1930-1937 (2001).

15. Stasiek, J. A. \& Kowalewski, T. A. Thermochromic liquid crystals applied for heat transfer research. Opto-Electron. Rev. 10, 1-10 (2002).

16. Foe, V. E., Odell, G. M. \& Edgar, B. A. in The Development of Drosophila melanogaster (eds Bate, M. \& Martinez Arias, A.) (Cold Spring Harbor Press, New York, 1993).

17. Niemuth, J. \& Wolf, R. Developmental asynchrony caused by steep temperature-gradients does not impair pattern-formation in the wasp, Pimpla turionellae L. Roux's Arch. Dev. Biol. 204, 444-452 (1995).

18. Frasch, M. et al. Characterization and localization of the Even-skipped protein of Drosophila. EMBO J 6, 749-759 (1987)

19. Myasnikova, E. et al. Registration of the expression patterns of Drosophila segmentation genes by two independent methods. Bioinformatics 17, 3-12 (2001).

20. Patel, N. H., Condron, B. G. \& Zinn, K. Pair-rule expression patterns of Even-skipped are found in both short-germ and long-germ beetles. Nature 367, 429-434 (1994).

21. Witkowski, F. X. et al. Spatiotemporal evolution of ventricular fibrillation. Nature 392, 78-82 (1998).

Supplementary Information accompanies the paper on www.nature.com/nature.

Acknowledgements This work was supported by the Searle Scholars Program and was performed at the Chicago MRSEC microfluidic facility funded by the NSF. N.H.P. is an Investigator of the Howard Hughes Medical Institute. We thank J. B. Brokaw, C. A. Macrander and M. Giorgianni for preliminary experiments. We thank D. Bilder and I. Hariharan for discussions and comments on the manuscript.

Competing interests statement The authors declare that they have no competing financial interests.

Correspondence and requests for materials should be addressed to R.F.I. (r-ismagilov@uchicago.edu) and N.H.P. (nipam@uclink.berkeley.edu). pro-inflammatory gene promoters. Inactivation of IKK $\alpha$ in mice enhances inflammation and bacterial clearance. Hence, the two IKK catalytic subunits have evolved opposing but complimentary roles needed for the intricate control of inflammation and innate immunity.

NF- $\mathrm{B}$ transcription factors are pivotal regulators of inflammation and immunity that control expression of important immunoregulatory genes ${ }^{2,3}$. NF- $\kappa \mathrm{B}$ activation and activity are tightly controlled by a number of endogenous mechanisms that limit the excessive and prolonged production of pro-inflammatory mediators, which can cause tissue damage during the inflammatory response $^{3,4}$. With the exception of autoregulated Ік $\mathrm{B} \alpha$ (inhibitor of NF-kB alpha) expression ${ }^{4}$ and induction of the de-ubiquitinating enzyme A20 (ref. 5), the mechanisms that limit the duration and magnitude of NF-кB signalling are poorly understood. It is likely that $\mathrm{I \kappa} \mathrm{B} \alpha$ and $\mathrm{A} 20$ are not the only physiologically relevant negative regulators of this central signalling module. The activation of NF- $\mathrm{B}$ by pro-inflammatory stimuli depends on the classical IKK complex, composed of two catalytic subunits (IKK $\alpha$ and IKK $\beta$ ), together with a regulatory subunit IKK $\gamma / \mathrm{NEMO}^{3,6}$. IKK activation is triggered by engagement of cytokine receptors as well as pattern recognition receptors. Gene disruption studies revealed that in addition to IKK $\gamma / \mathrm{NEMO}$, which is necessary for activation of the classical IKK complex ${ }^{7}$, it is IKK $\beta$ rather than IKK $\alpha$ that plays a more critical role in activating inflammation ${ }^{6}$. IKK $\alpha$ forms an alternative complex (without IKK $\beta$ and IKK $\gamma$ ) $^{8}$, the function of which is required for lymphoid organ development and B cell maturation ${ }^{9}$. This alternative signalling pathway is activated by certain members of the tumour necrosis factor (TNF) family, but not by pattern recognition receptors such as Toll-like receptor 4 (TLR4) (ref. 2). The function of IKK $\alpha$ within the classical IKK complex, however, is not entirely clear. Although a chromatinmodifying function for IKK $\alpha$ required for TNF $\alpha$-mediated gene induction has been suggested ${ }^{10,11}$, targeting of the $I k k \alpha$ (also called Chuk) gene in mice does not support this proposal ${ }^{2}$.

We investigated the role of IKK $\alpha$ in inflammation and innate immunity in vivo, using mice that express the inactivatable variant $\mathrm{IKK} \alpha(\mathrm{AA})$ (ref. 9). I $k k \alpha^{A A / A A}$ mice (which are homozygous for the mutant allele) and littermate controls were challenged systemically with the Gram-positive human pathogen group B Streptococcus $(\mathrm{GBS})^{12}$, and monitored for bacterial clearance and survival. Although $I k k \alpha^{A A / A A}$ mice showed significantly decreased blood bacterial counts at $24 \mathrm{~h}$ (Fig. 1a), mortality was notably accelerated relative to wild-type animals (Fig. 1b). This paradoxical result suggested that $I k k \alpha^{A A / A A}$ mice have an exacerbated inflammatory response to infection that enhances bacterial clearance but provokes septic shock. To pursue this hypothesis further, we assessed the local inflammatory response to bacterial infection in a non-lethal model. When $I k k \alpha^{A A / A A}$ mice and littermate controls were inoculated intranasally with GBS, the mutants showed increased bacterial clearance, associated with increased neutrophil recruitment and local inflammation (Fig. 1c-e). Together, these studies indicate that $\mathrm{IKK} \alpha$ is somehow involved in limiting the inflammatory response to Gram-positive infection.

We extended these studies to a model of Gram-negative septic shock by using the TLR4 agonist lipopolysaccharide (LPS) from Escherichia coli $^{13}$. I $k k^{A A / A A}$ mice showed increased susceptibility to LPS-induced septic shock (Fig. 2a). Real-time quantitative polymerase chain reaction (PCR) analysis of liver and lung RNA showed elevated expression of pro-inflammatory and antiapoptotic NF-кB target genes, including macrophage inflammatory protein (MIP)-2, MIP-1 $\alpha$, interleukin (IL)-12p40, inhibitor of apoptosis protein (IAP)-2 and inducible nitric oxide synthase (iNOS) (Fig. 2b). Local LPS administration to the lung also resulted in elevated pulmonary leukocyte recruitment and proinflammatory cytokine production in $I k k^{A A / A A}$ mice relative to wild-type littermates (see Supplementary Fig. 1). Neutrophil 